\title{
CÁC PHƯƠNG THỨC NHậT HÓA TÙ̉ NGOẠI LAI GỐC TIẾNG ANH TRONG TIẾNG NHẬT (NHİN TƯ GÓC ĐỘ NGỮ ÂM)
}

\author{
Ngô Minh Thủy*, Trần Kiều Huế \\ Khoa Ngôn ngũ và Văn hóa Nhật Bản, Trưòng Đại học Ngoại ngũu, ĐHQGHN, \\ Phạm Văn Đồng, Cầu Giấy, Hà Nội, Việt Nam \\ Nhận bài ngày 09 tháng 12 năm 2016 \\ Chỉnh sửa ngày 09 tháng 01 năm 2017; Chấp nhận đăng ngày 10 tháng 01 năm 2017 \\ Tóm tắt: Đối với những người nước ngoài học tiếng Nhật, đặc biệt là những người biết tiếng Anh, \\ nhóm từ ngoại lai gốc tiếng Anh trong tiếng Nhật là nhóm từ khó, bởi vì các từ tiếng Anh khi nhập vào tiếng \\ Nhật đều có những sự thay đổi lớn, đặc biệt là về ngữ âm và chữ viết, nhiều khi tới mức "khó nhận ra" ngay \\ đối với người bản ngữ nói tiếng Anh. Trong bài viết này, trên cơ sở khái quát những đặc điểm cơ bản của ngữ \\ âm, chữ viết tiếng Nhật và những điểm khác biệt cơ bản của ngữ âm tiếng Anh so với ngữ âm tiếng Nhật, \\ các tác giả phân tích các phương thức "Nhật hóa" nhóm từ ngoại lai gốc tiếng Anh trong tiếng Nhật từ góc \\ độ ngữ âm, nhằm giúp người học tiếng Nhật nắm được một số quy tắc Nhật hóa từ tiếng Anh và dễ dàng hơn \\ trong việc nhận biết và sử dụng nhóm từ ngoại lai gốc tiếng Anh trong tiếng Nhật.
}

Tù khóa: từ ngoại lai, từ ngoại lai gốc tiếng Anh, phương thức Nhật hóa, ngữ âm

Nhìn từ góc độ nguồn gốc của từ, các nhà Nhật ngữ học chia từ vựng tiếng Nhật ra thành 3 nhóm lớn, gồm: từ thuần Nhật, từ Hán và từ ngoại lai. Từ thuần Nhật (Wago, hay còn gọi là Yamato kotoba) là những từ được sử dụng ở Nhật Bản từ trước khi có sự du nhập của các từ Hán (mặc dù trong lớp từ được gọi là từ thuần Nhật cũng có những từ du nhập từ tiếng Hán cổ, tiếng Triều Tiên hoặc từ ngôn ngữ Ainu, nhưng không có căn cứ chắc chắn để kết luận là từ ngoại lai, và người Nhật Bản cũng không còn coi là từ ngoại lai (ví dụ: てら (寺) tera: chùa,うま（馬）uma: con ngựa, うなぎ（ 鰻） unagi: con lươn...). Từ Hán là từ du nhập từ tiếng Hán vào tiếng Nhật, được viết bằng chữ Hán và đọc theo âm Hán đã được Nhật hoá (ví dụ: 意見iken: ý kiến, 記念kinen: kỷ niệm). Từ ngoại lai là những từ du nhập vào tiếng Nhật từ các ngôn ngữ khác ngoài tiếng Hán (chủ yếu là

\footnotetext{
* Tác giả liên hệ. ĐT.: 84-904139936
}

Email:ngothuyvn@vnu.edu.vn các ngôn ngữ Châu Âu), đã được Nhật hoá về hình thức và ý nghĩa (ví dụ: picnic [piknik] $\rightarrow$ ピクニック [pikuniQku], topic [tว pik] $\rightarrow$ ト ピック [topiQku]). Ngoài ra, còn có nhóm thứ 4 là các từ có cấu tạo hỗn hợp từ ba loại trên, ví dụ: 電子メール (thư điện tử) là sự kết hợp giữa từ 電子(denshi: điện tử - từ Hán) và từ メ 一ル(meeru: thư - từ tiếng Anh), 一人つ子政 策 (chính sách một con, trong đó 一人つ子là yếu tố thuần Nhật, 政策là yếu tố Hán.)

Năm 1966, Viện Nghiên cứu Quốc gia về quốc ngữ của Nhật Bản tiến hành khảo sát từ vựng trong số báo ra buổi chiều của ba tờ báo lớn（『朝日』- Asahi,『毎日』 - Mainichi, 『読売』 - Yomiuri). Kết quả thu được liên quan đến tỉ lệ sử dụng từ vựng nhìn từ nguồn gốc của từ như sau: từ ngoại lai chiếm $12 \%$, từ thuần Nhật: $38,8 \%$, từ Hán: 44,3\%, từ hỗn hợp: 4,8\%. Tiếp theo đó, một cuộc khảo sát tương tự được thực hiện đối với văn nói (ghi âm các cuộc nói chuyện của nhóm người sống 
ở Tokyo và vùng ngoại $\hat{o}$, thời lượng là 42 tiếng đồng hồ) đưa ra kết quả về tỷ lệ xuất hiện của 4 nhóm từ vựng này lần lượt như sau: từ ngoại lai chiếm $10,0 \%$; từ thuần Nhật: 46,9\%; từ Hán: 40,0\%; từ hỗn hợp: 3,0\%. Riêng trong nhóm từ ngoại lai, nếu phân tích theo nguồn gốc của từ, theo kết quả thống kê do Viện Nghiên cứu Quốc gia về quốc ngữ của Nhật Bản thực hiện, thì các từ ngoại lai có nguồn gốc tiếng Anh có số lượng áp đảo, với 2.395 từ trong tổng số 2.964 từ ngoại lai xuất hiện trên 90 tạp chí hiện đại của Nhật Bản (dẫn theo Ngô Minh Thuỷ 2005, Tù ngoại lai trong tiếng Nhật). Cụ thể như sau:

Bảng 1. Tỷ lệ xuất hiện của từ ngoại lai trong tiếng Nhật tính theo nguồn gốc ngôn ngữ

\begin{tabular}{|c|c|c|c|c|c|}
\hline $\begin{array}{l}\text { Tiếng } \\
\text { Anh: } \\
2.395\end{array}$ & $\begin{array}{c}\text { Tiếng } \\
\text { Đức: } \\
99\end{array}$ & $\begin{array}{c}\text { Tiếng } \\
\text { Italy: } \\
44\end{array}$ & $\begin{array}{l}\text { Tiếng } \\
\text { Nga: } \\
25\end{array}$ & $\begin{array}{c}\text { Tiếng Tây } \\
\text { Ban Nha: } \\
21\end{array}$ & $\begin{array}{l}\text { Các } \\
\text { tiếng } \\
\text { khác: } \\
114\end{array}$ \\
\hline $\begin{array}{c}\text { Tiếng } \\
\text { Pháp: } \\
166\end{array}$ & $\begin{array}{c}\text { Tiếng } \\
\text { Trung } \\
\text { Quốc: } \\
22\end{array}$ & $\begin{array}{l}\text { Tiếng } \\
\text { Hà } \\
\text { Lan: } \\
40\end{array}$ & $\begin{array}{c}\text { Tiếng } \\
\text { Bồ Đào } \\
\text { Nha: } \\
21\end{array}$ & $\begin{array}{c}\text { Tiếng } \\
\text { Latinh: } 15\end{array}$ & \\
\hline
\end{tabular}

Tiếng Nhật có một số lượng lớn từ ngoại lai gốc tiếng Anh như vậy, nhưng có thể nói rằng đối với những người nước ngoài học tiếng Nhật, đặc biệt là những người biết tiếng Anh, nhóm từ ngoại lai gốc tiếng Anh này lại gây không ít khó khăn, bởi các từ tiếng Anh khi nhập vào tiếng Nhật đều có những sự thay đổi lớn, đặc biệt là về ngữ âm và chữ viết, nhiều khi tới mức "khó nhận ra" ngay đối với người bản ngữ nói tiếng Anh. Chính vì lẽ đó, trong bài viết này, trên cơ sở khái quát những đặc điểm cơ bản của ngữ âm, chữ viết tiếng Nhật và những điểm khác biệt cơ bản của ngữ âm tiếng Anh so với ngữ âm tiếng Nhật, chúng tôi tiến hành phân tích các phương thức "Nhật hóa" nhóm từ tiếng Anh trong tiếng Nhật từ góc độ ngữ âm, nhằm giúp người học tiếng Nhật nắm được một số quy tắc Nhật hóa từ tiếng Anh và dễ dàng hơn trong việc nhận biết và sử dụng nhóm từ ngoại lai gốc tiếng Anh trong tiếng Nhật.

\section{Một số đặc điểm của ngữ âm của tiếng Nhật và những điểm khác biệt cơ bản của ngữ âm tiếng Anh so với ngữ âm tiếng Nhật}

\subsection{Một số đặc điểm của ngũ âm tiếng Nhật}

\subsubsection{Phách trong tiếng Nhật}

Phách (còn gọi là mora) được coi là đơn vị phát âm nhỏ nhất trong tiếng Nhật và có thể coi đây là đặc trưng quan trọng của ngữ âm tiếng Nhật. Ví dụ, khi phát âm từ [nihoNyo] (にほんご- 日本語, nihongo - có nghĩa là "tiếng Nhật"), thì người Nhật chia từ này thành bốn "đoạn" âm với độ dài bằng nhau là

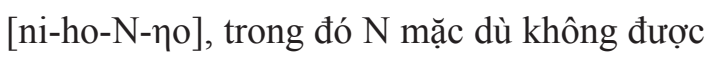
phát âm rõ ra thành tiếng nhưng vẫn "chiếm" một “đoạn”, tương đương một phách khi phát âm. Người Nhật cho rằng từng phần, từng đoạn như thế là đơn vị nhỏ nhất của âm thanh, gọi là phách. Có thể hình dung phách như đơn vị phách trong âm nhạc. Và như vậy, từ に ほんご(日本語, nihongo) nêu trên là tập hợp của 4 phách. Trong từ này, loại trừ âm $[\mathrm{N}]$ (âm mũi), còn 3 đơn vị phát âm còn lại ( 3 phách còn lại) là kết hợp của một phụ âm với một nguyên âm: に $[\mathrm{n}+\mathrm{i}]$, ほ $[\mathrm{h}+\mathrm{o}]$, ご$[\eta+\mathrm{o}]$.

Nếu theo quan điểm thông thường về âm tiết ("âm tiết phải có tính vang; mỗi âm tiết phải có một bộ phận hạt nhân gồm ít nhất một nguyên âm" - Hyman, 1975, 189. Dẫn theo Đoàn Xuân Kiên, 1998) thì có thể thấy rằng phách trong tiếng Nhật không hoàn toàn trùng với khái niệm âm tiết trong các ngôn ngữ khác, và như vậy, có thể nói rằng trong tiếng Nhật đồng thời tồn tại khái niệm âm tiết và khái niệm phách, và hai khái niệm này không trùng với nhau (Mc Cawley, 1968). Hãy thử so sánh số 
lượng âm tiết và số lượng phách trong các từ dưới đây:

$$
\begin{array}{ll}
\text { さんぽ（tản bộ) } & \text { きっぷ (vé) } \\
\text { [san po] } 2 \text { âm tiết } & \text { [kiQp pu] } 2 \text { âm tiết } \\
\text { / sa N po/ } 3 \text { phách } & \text { / ki Qp pu/3 phách }
\end{array}
$$

thành tiếng) tương đương như một âm tiết thông thường nêu trên. Nói cách khác, khi phách (hay

Như vậy, theo như phân tích ở trên, số lượng phách trong 1 từ tiếng Nhật có thể bằng số lượng âm tiết, hoặc là nhiều hơn số lượng âm tiết trong từ đó.

\subsection{2. Âm tiết trong tiếng Nhật}

Về cơ bản, cấu trúc âm tiết của tiếng Nhật là âm tiết mở (opended syllable, tức là âm tiết có kết thúc là một nguyên âm), ví dụ: はな [ha na] bông hoa), $く<[i \mathrm{ku}]$ - đi, うそ [u so]- điều nói dối). Nếu quy định $\mathrm{C}$ là phụ âm, $\mathrm{S}$ là bán nguyên âm, $\mathrm{V}$ là nguyên âm thì âm tiết tiếng Nhật thông thường có các dạng cấu trúc như sau:

V: ví dụ: ア ア $\mathrm{a}] 、 イ[\mathrm{i}] 、 ウ[\mathrm{u}] 、 エ$ $[\mathrm{e}] 、 才[\mathrm{o}]$

CV: ví dụ: カ $[\mathrm{ka}] 、 キ[\mathrm{ki}] 、 ク[\mathrm{ku}]$ 、 ケ $[\mathrm{ke}] 、 \sqsupset[\mathrm{ko}]$

CSV: ví dụ: キャ[kja]、キュ[kju]、キ $\exists[\mathrm{kjo}]$

Ngoài các âm tiết thông thường có các cấu trúc như nêu trên và có độ dài phát âm tương đương với 1 phách (mora), như ở phần trên đã nói, trong tiếng Nhật còn có một số âm đặc thù cũng có độ dài tương đương với độ dài của 1 phách khi phát âm. Đó là các âm mũi (được biểu thị bằng $/ \mathrm{N} /$ ) và âm ngắt (được biểu thị bằng /Q/). Ngoài ra, còn có bán nguyên âm dài [V:], có nghĩa là thêm một âm [:] kéo dài ngay sau nguyên âm thông thường, và âm kéo dài này cũng được coi là âm đặc thù. Các âm đặc thù này khác với các âm thông thường khác là không bao giờ xuất hiện ở đầu từ. Khi phát âm, các âm đặc thù này được tính là một phách, có độ dài (không phát mora) được thể hiện bằng các âm đặc biệt nêu trên, thì phách/ mora đó chính là 1 bộ phận trong âm tiết của từ. Ví dụ: từ「ピッチャー」 : gồm 4 phách (4 mora), 2 âm tiết, trong đó âm tiết thứ nhất bao gồm âm ngắt/Q/ (ツ), âm tiết thứ 2 bao gồm âm dài [V:] (一).

Như vậy, cấu trúc âm tiết của tiếng Nhật được thể hiện như sau:

$$
\text { ( C (S) ) V (:) }\{\mathbf{N}, \mathbf{Q}\}
$$

Trong công thức nêu trên, $\mathrm{C}$ là phụ âm, $\mathrm{S}$ là bán nguyên âm $[\mathrm{j}]$ hoặc $[\mathrm{w}], \mathrm{V}$ là nguyên âm, $\mathrm{N}$ là âm mũi, Q là âm ngắt, [ : ] là âm kéo dài; ký hiệu ( ) trong công thức trên thể hiện yếu tố không bắt

\begin{tabular}{|c|c|c|c|c|c|}
\hline \multicolumn{3}{|c|}{$\begin{array}{l}\text { Ví dụ về các âm tiết có } \\
\text { nòng cốt là nguyên âm } \\
\text { ngắn }\end{array}$} & \multicolumn{3}{|c|}{$\begin{array}{l}\text { Ví dụ về các âm tiết có nòng } \\
\text { cốt là nguyên âm dài }\end{array}$} \\
\hline $\mathrm{V}$ & $\mathrm{a}$ & あ & $\mathrm{V}:$ & $\mathrm{u}:$ & うう \\
\hline $\mathrm{CV}$ & $\mathrm{ka}$ & か & $\mathrm{CV}:$ & $\mathrm{ku}:$ & $<う$ \\
\hline SV & ja & や & SV: & yu: & ゆう \\
\hline CSV & kja & きや & CSV: & kju: & きゆう \\
\hline $\mathrm{VN}$ & $\mathrm{aN}$ & あん & CV:N & $\mathrm{ku}: \mathrm{N}$ & \\
\hline $\mathrm{CVN}$ & $\mathrm{kaN}$ & かん & V:N & $\mathrm{u}: \mathrm{N}$ & ううん \\
\hline $\mathrm{VQ}(\mathrm{CV})$ & $\mathrm{aQ}($ te $)$ & あって & *V:Q (CV) & $\mathrm{u}: \mathrm{Q}$ (to) & $\begin{array}{l}\text { ううつ } \\
\text { (と) }\end{array}$ \\
\hline $\mathrm{CVQ}(\mathrm{CV})$ & $\mathrm{kaQ}(\mathrm{te})$ & かって & *CV:Q(CV) & $\mathrm{Ku}: \mathrm{Q}$ (to) & $\begin{array}{c}\text { ずうつ } \\
\text { と }\end{array}$ \\
\hline
\end{tabular}
buộc; \{\} biểu thị yếu tố có thể lựa chọn.

Dưới đây là một số ví dụ về các âm tiết được biểu thị bởi công thức trên, và đây cũng là các âm tiết xuất hiện ở các từ thuần Nhật.

Bảng 2. Các ví dụ về loại âm tiết thường xuất hiện trong các từ tiếng Nhật

Ghi chú: Trong bảng nêu trên, ký hiệu * thể hiện ý "chủ yếu xuất hiện trong các tù tuoơng thanh, tuoơng hình”; ki hiệu ( ) chi các yếu tố không bắt buộc. 


\subsection{Chũu viết trong tiếng Nhật và Bảng 50 âm}

Tiếng Nhật sử dụng 3 hệ thống chữ viết gồm: chữ Hiragana, chữ Katakana và chữ Hán. Chữ Hiragana và chữ Katakana được gọi chung là chữ Kana, là hệ thống chữ Nhật (trong đó chữ Katakana dùng để viết các từ ngoại lai hoặc tên riêng nước ngoài). Ngoài ra, trong tiếng Nhật còn sử dụng chữ Latin (Romaji) để viết cách đọc các từ tiếng Nhật cho dễ đọc đối với người nước ngoài.

Chữ Kana được thể hiện qua "Bảng 50 âm” bằng chữ Hiragana hoặc Katakana. Dưới đây là các âm trong tiếng Nhật thể hiện trong Bảng 50 âm viết bằng chữ Katakana.

Bảng 3. Hệ thống các âm trong tiếng Nhật điều này ảnh hưởng không nhỏ đối với việc Nhật hoá nguyên âm tiếng Anh (chúng tôi sẽ trình bày cụ thể trong phần sau).

Trong tiếng Anh có khá nhiều phụ âm kép. Trong tiếng Nhật cũng tồn tại phụ âm kép nhưng số lượng rất ít, chủ yếu là biến thể phát âm của phụ âm [z], [z] xuất hiện tuỳ thuộc vào vị trí của phụ âm đó trong tổ hợp âm tiết (đầu, giữa, cuối) và không có sự phân biệt về mặt chữ viết. Tiếng Anh có số lượng phụ âm cuối nhiều, trong khi đó tiếng Nhật có phụ âm cuối duy nhất là âm mũi $ん$ [N].

Khác với tiếng Nhật có cấu trúc âm tiết cơ bản là âm tiết mở, cấu trúc âm tiết phổ biến của tiếng Anh là âm tiết đóng (closed syllable, tức là âm tiết có kết thúc là phụ âm, ví dụ "hot"

\begin{tabular}{|c|c|c|}
\hline & Trực âm (được viết bằng 1 chữ Kana) & Âm kép (được viết bằng 2 chữ Kana) \\
\hline $\begin{array}{l}\text { Thanh âm } \\
\text { (Âm trong) }\end{array}$ & 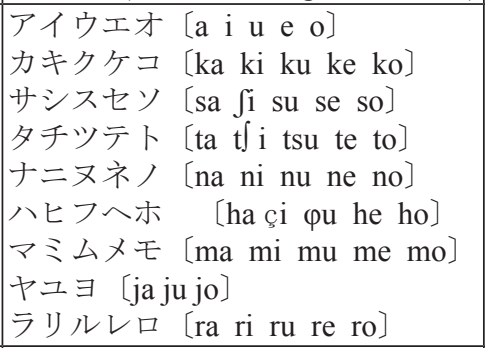 & 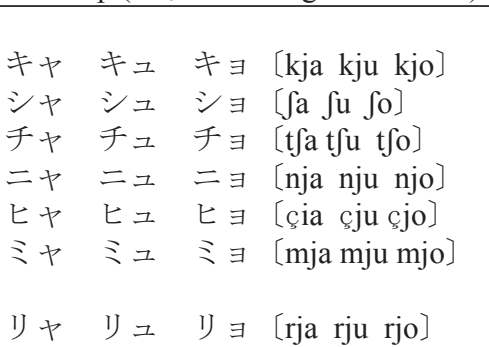 \\
\hline $\begin{array}{l}\text { Đục âm } \\
\text { (Âm đục) }\end{array}$ & 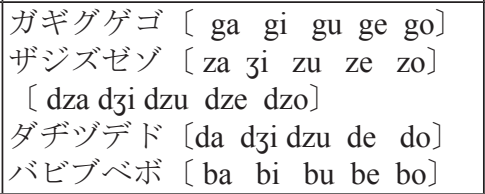 & 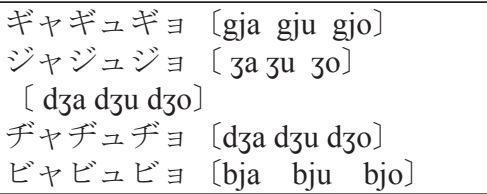 \\
\hline $\begin{array}{l}\text { Bán đục âm } \\
\text { (Âm bán đục) }\end{array}$ & イ゚゚〔pa pi pu pe po] & ピヤピュピヨ [pja pju pjo $]$ \\
\hline
\end{tabular}

\subsection{Nhũng điểm khác biệt co bản của ngũ âm} tiếng Anh so với ngũ âm tiếng Nhật

Khác với tiếng Nhật (sử dụng 3 loại chữ: chữ Hiragana, chữ Katakana và chữ Hán), tiếng Anh sử dụng 1 loại chữ viết thuộc hệ chữ Latin.

Tiếng Anh có 24 phụ âm và 20 nguyên âm (tương đối nhiều so với tiếng Nhật chỉ có 5 nguyên âm), trong đó có 12 nguyên âm đơn và 8 nguyên âm đôi. Sự khác nhau về nguyên âm so với tiếng Nhật không chỉ đơn thuần ở mặt số lượng mà còn ở tính chất của nguyên âm, [hot], "kick" [kik], "some" [รəm]...). Trong tiếng Anh có nhiều trường hợp xuất hiện các biến thể phát âm của một nguyên âm hoặc phụ âm do vị trí tham gia khác nhau của các âm này trong âm tiết, nhưng trong tiếng Nhật thì hầu như không có hiện tượng này, ngoại trừ một số trường hợp liên quan đến các âm tiết đặc thù (âm ngắt, âm mũi, âm dài).

Những điểm khác nhau nêu trên có ảnh hưởng rất lớn đến việc Nhật hóa các từ tiếng Anh trong tiếng Nhật về mặt ngữ âm. 
2. Các phương thức Nhật hoá từ tiếng Anh về mặt ngữ âm

Với các đặc điểm ngữ âm và chữ viết khác nhau giữa tiếng Anh và tiếng Nhật như đã nêu ở trên, khi được "tiếp nhận" vào tiếng Nhật, các từ tiếng Anh đã được Nhật hóa theo các phương thức khác nhau về mặt ngữ âm. Có 6 phương thức cơ bản trong việc Nhật hóa từ tiếng Anh về mặt ngữ âm, gồm:

1) Bổ sung nguyên âm (chuyển đổi thành âm tiết mở);

2) Chèn thêm âm ngắt;

3) Nhật hoá nguyên âm;

4) Nhật hóa phụ âm và bán nguyên âm;

5) Nhật hóa theo cách đánh vần;

6) Nhật hóa trọng âm.

Với mỗi từng phương thức Nhật hóa có những nguyên tắc chung và những trường hợp ngoại lệ riêng. Dưới đây là những nội dung cơ bản của 6 phương thức Nhật hóa này.

\subsection{Bổ sung nguyên âm (chuyển đổi thành âm tiết mở)}

Hiện tượng bổ sung nguyên âm chủ yếu là do sự khác nhau về cấu tạo âm giữa tiếng Nhật và tiếng Anh.

Nếu lược bỏ các trường hợp có chứa bán nguyên âm, trong tiếng Anh có các kiểu âm tiết sau: V, CV, VC, CVC (C: phụ âm, V: nguyên âm). Có thể thấy rằng tiếng $A n h$ có rất nhiều âm tiết kết thúc bằng phụ âm và không hiếm các âm tiết có các phụ âm đứng liền nhau ở phía trước hoặc phía sau nguyên âm. Ví dụ:

$$
\begin{aligned}
& \text { spring [s } \left.\begin{array}{llllll}
s & \mathrm{p} & \mathrm{r} & \mathrm{i} & \mathrm{n} & \mathrm{g}
\end{array}\right] \\
& \begin{array}{llllll}
\mathrm{C} 1 & \mathrm{C} 2 & \mathrm{C} 3 & \mathrm{~V} & \mathrm{C} 4 & \mathrm{C} 5
\end{array}
\end{aligned}
$$

Trong khi đó, tiếng Nhật chỉ có 2 âm tố tạo nên âm tiết đóng là âm mũi $/ \mathrm{N} /$ và âm ngắt /Q/ và có sự hạn chế đối với các loại phụ âm đi sau âm ngắt /Q/. Ngoài ra, trong tiếng Nhật không xuất hiện một nhóm các phụ âm liền nhau giống như tiếng Anh. Chính vì vậy, khi Nhật hoá các từ tiếng Anh, trừ trường hợp phần đuôi của âm tiết được Nhật hoá và trở thành âm mũi $/ \mathrm{N} /$, còn lại các trường hợp khác phải chuyển âm tiết tiếng Anh thành âm tiết mở, tức là bổ sung nguyên âm vào sau phụ âm hoặc chèn thêm nguyên âm vào giữa hai phụ âm.

Ví dụ, nếu chuyển từ "Christmas' sang tiếng Nhật thì phải bổ sung nguyên âm vào phía sau các phụ âm trong tiếng Anh và từ này trở thành từ có 5 âm tiết trong tiếng Nhật: ク リスマス (ku-ri-su-ma-su), từ này có cấu trúc âm tiết là:

\section{ku-ri-su: C1V1- C2V2- C3V3}

-ma-su: C4V4 - C5V5 (C: phụ âm, V: nguyên âm)

Tất cả các nguyên âm trong tiếng Nhật có thể được sử dụng để bổ sung trong các trường hợp này, nhưng sử dụng nguyên âm nào thì phần lớn tuỳ thuộc vào các phụ âm, có nghĩa là có qui tắc trong việc chuyển đổi các từ tiếng Anh sang tiếng Nhật đối với hầu hết trường hợp sử dụng các nguyên âm bổ sung cho các phụ âm. Dưới đây là một số phương thức bổ sung nguyên âm vào từ gốc tiếng Anh, hay còn gọi là Nhật hóa âm tiết tiếng Anh.

Bảng 4. Một số kiểu Nhật hoá âm tiết tiếng Anh

\begin{tabular}{|c|c|c|c|}
\hline $\begin{array}{c}\text { Cấu trúc } \\
\text { âm tiết } \\
\text { của từ } \\
\text { trong } \\
\text { tiếng Anh }\end{array}$ & $\begin{array}{c}\text { Cấu trúc } \\
\text { âm tiết } \\
\text { của từ } \\
\text { đã được } \\
\text { Nhật hoá }\end{array}$ & $\begin{array}{c}\text { Ví dụ } \\
\text { (tiếng } \\
\text { Anh) }\end{array}$ & $\begin{array}{c}\text { Ví dụ } \\
\text { (từ đã } \\
\text { được Nhật } \\
\text { hóa })\end{array}$ \\
\hline CVC & C V C V & $\begin{array}{c}\text { mass } \\
\text { [mas] }\end{array}$ & [ma su] \\
\hline CCVC & $\begin{array}{c}\text { C V C V } \\
\text { C V }\end{array}$ & $\begin{array}{c}\text { breath } \\
\text { [bre@] }\end{array}$ & [buresu] \\
\hline CCCVC & $\begin{array}{c}\text { C V C V C } \\
\text { V C V }\end{array}$ & $\begin{array}{c}\text { stress } \\
\text { [stres] }\end{array}$ & [sutoresu] \\
\hline CVCC & CVCVCV & best [best] & [besuto] \\
\hline CVCCC & $\begin{array}{c}\text { CVCV } \\
\text { CVCV }\end{array}$ & $\begin{array}{c}\text { pickles } \\
\text { [pick] }\end{array}$ & [pikurusu] \\
\hline
\end{tabular}

Các phụ âm [t, d] của tiếng Anh khi chuyển sang tiếng Nhật thường bổ sung 
nguyên âm [o], cũng có trường hợp sử dụng nguyên âm [u]. Tuy nhiên, [o] được sử dụng nhiều hơn hẳn so với các trường hợp dùng 〔u〕 , ví dụ như các từ sau: アウト ライン(outline),アドレッス(adress); 〔u] chỉ được thấy xuất hiện nhiều trong các từ cũ trước đây. Trái lại, các phụ âm 〔k, g \hì phổ biến là bổ sung 〔u〕 ví dụ như: マークシー ト (marksheet), ハンドバッグ(handbag), マ スク (maske), rất hiếm khi dùng 〔o〕.

Ngoài ra, cũng có một số trường hợp thêm nguyên âm 〔i \ vào sau phụ âm 〔k〕 , ví dụ như ストライキ (strike: biểu tình). Tuy nhiên, có thể thấy rằng trường hợp này là do chịu ảnh hưởng âm sắc của nguyên âm đứng gần nó ( 〔e〕).

Thông thường khi chuyển sang tiếng Nhật, phụ âm 〔t] của tiếng Anh sẽ được phát âm thành [to ] (“ト”), [k] sẽ thành [ku] (“ク”). Còn các trường hợp khác $〔 \mathrm{k}$, $\mathrm{s}, \mathrm{f}, \mathrm{r}, \mathrm{z}, \mathrm{m}, \mathrm{b}$ ] đều ghép thêm nguyên âm [i] vào sau các phụ âm này, ví dụ như "table" $\rightarrow$ te: $\underline{\text { buru }}$, "ball” $\rightarrow$ bo: $\underline{\text { ru }}$, "rhythm" $\rightarrow$ rizumu,

Trong tiếng Nhật hiện đại, loại trừ hàng “タ” [ta], còn lại các hàng khác đều có xu hướng bổ sung thêm nguyên âm "u" (theo cột “ウ” [u] ). Ngoài ra, trường hợp các âm tiết có [r] đi sau nguyên âm trong từ gốc tiếng Anh, khi sang tiếng Nhật có thể [r] sẽ mất đi và trở thành âm tiết có kết thúc bằng nguyên âm dài, vì vậy trường hợp này không phải bổ sung hay chèn thêm nguyên âm. Ví dụ như “corner" $\rightarrow$ ユーナー [ko : na :] hoặc "super" $\rightarrow$ スーパー $[\mathrm{su}: \mathrm{pa}:]$

Có thể diễn đạt các nguyên tắc trên qua các ví dụ trong Bảng dưới đây.
Bảng 5. Một số ví dụ về việc bổ sung nguyên âm tương ứng

\begin{tabular}{|c|c|c|}
\hline $\begin{array}{l}\text { Phụ âm } \\
\text { tiếng Nhật }\end{array}$ & $\begin{array}{l}\text { Các nguyên } \\
\text { âm bô̂ sung }\end{array}$ & Ví dụ \\
\hline $\mathrm{t}, \mathrm{d}$ & o & 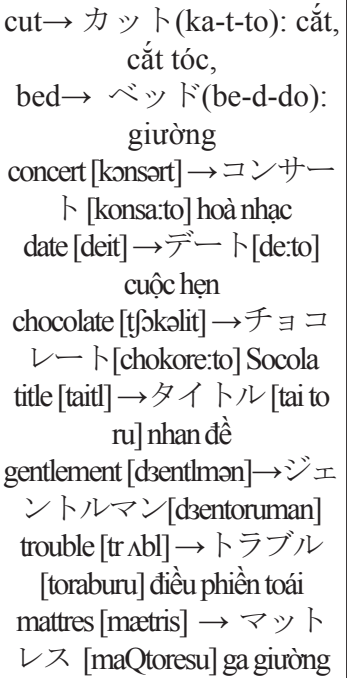 \\
\hline ch, ge & $\mathrm{i}$ & $\begin{array}{c}\text { research } \rightarrow \text { リサーチ(ri- } \\
\text { sa:chi): nghiên cứu, } \\
\text { stage } \rightarrow \text { ステージ(su-te- } \\
\text { dgi:): sân khấu, giai đoạn }\end{array}$ \\
\hline $\begin{array}{c}\text { Các phụ âm } \\
\text { khác }\end{array}$ & $\mathrm{u}$ & $\begin{array}{c}\text { cup } \rightarrow \text { カップ(ka-p-pu): } \\
\text { cốc, cup } \\
\text { tool } \rightarrow \text { ツール(tsu:ru) : } \\
\text { công cụ } \\
\text { goods } \rightarrow \text { グッズ (gu-d- } \\
\text { zu): hàng hoá }\end{array}$ \\
\hline
\end{tabular}

\subsection{Chèn thêm âm ngắt}

Âm ngắt trong tiếng Nhật được gọi là 促 音 [sokuon] hay (つまる音) [tsumaru oto]， cùng với phụ âm liền sau nó tạo thành phụ âm kép. Khi phát âm, âm ngắt của tiếng Nhật tạo ra sự căng của thanh hầu và có độ dài thời gian bằng với các phách khác. Khi Nhật hóa từ tiếng Anh, âm ngắt (kí hiệu trong phiên âm là $[\mathrm{Q}])$ được chèn vào giữa nguyên âm và phụ âm. Điều kiện để thực hiện thao tác này là các nguyên âm [i, e, æ, $\Lambda, \curvearrowright, u]$ trong tiếng Anh cũng là các nguyên âm ngắn và tất cả phụ âm ở đuôi từ đều là phụ âm vô thanh và các âm đó phải là âm tắc $[\mathrm{p}, \mathrm{t}, \mathrm{k}]$, âm xát $\left[\mathrm{s}, \int\right]$ hoặc âm tắc xát [ts, tf]. Dưới đây là một số ví dụ: 
Bảng 6. Hiện tượng chèn thêm âm ngắt đối

với các từ một âm tiết

\begin{tabular}{|c|c|c|c|c|c|c|c|}
\hline \begin{tabular}{|l|} 
Âm cuối \\
của từ
\end{tabular} & $\sim p$ & $\sim \mathrm{t}$ & $\sim \mathrm{k}$ & $\sim$ ts & $\sim \mathrm{t} \int$ & $\sim \mathrm{S}$ & $\sim \int$ \\
\hline [i] & $\begin{array}{c}\operatorname{lip}[\operatorname{lip}\rfloor \\
\text { リップ }[\mathrm{riQpu}]\end{array}$ & $\begin{array}{c}\text { hit [hit] } \\
\text { ヒット } \\
\text { [hiQto] }\end{array}$ & $\begin{array}{c}\text { pick [pik } \\
\text { ピック } \\
{[\mathrm{piQku}]}\end{array}$ & - & $\begin{array}{c}\text { rich 〔rit〕〕 } \\
\text { リ?ッチ } \\
\left.\text { 〔riQt } \int i\right]\end{array}$ & $\begin{array}{c}\text { kiss [kis] } \\
\text { キッス } \\
\text { [kiQsu] }\end{array}$ & $\begin{array}{l}\text { fish 〔fij] } \\
\text { フィッシュ } \\
\text { 〔fiQfu } \int\end{array}$ \\
\hline$[\mathrm{e}]$ & $\begin{array}{l}\text { step 〔step〕 } \\
\text { ステップ } \\
\text { 〔steQpu〕 }\end{array}$ & $\begin{array}{l}\text { pet [pet] } \\
\text { ペット } \\
\text { [peQto }]\end{array}$ & $\begin{array}{c}\text { deck }[\text { dek }] \\
\text { デック } \\
{[\mathrm{deQku}]}\end{array}$ & - & $\begin{array}{l}\mathrm{H}[\mathrm{e} t \mathrm{t}] \\
\text { エッチ } \\
{\left[\mathrm{e} \mathrm{Qt} \int \mathrm{i}\right]}\end{array}$ & - & $\begin{array}{c}\left.\text { fresh } ₫ \text { fre } \int\right] \\
\text { フレッシュ } \\
\left.\text { [freQ } \int \mathrm{u}\right]\end{array}$ \\
\hline$[æ]$ & $\begin{array}{c}\text { map [mæp] } \\
\text { マップ } \\
{[\mathrm{maQpu}]}\end{array}$ & $\begin{array}{c}\text { hat [hæt] } \\
\text { ハット } \\
\text { [haQto] }\end{array}$ & $\begin{array}{c}\text { black [blæk] } \\
\text { ブラック } \\
\text { [braQku] }\end{array}$ & - & $\begin{array}{c}\left.\text { catch 〔kæt } \int\right] \\
\text { キャッチ } \\
{\left[\mathrm{kja} Q t \int \mathrm{i}\right]}\end{array}$ & - & $\begin{array}{c}\text { cash } \llbracket \mathrm{kæ} \int 〕 \\
\text { キャッシュ } \\
{\left[\mathrm{kjaQ} \int \mathrm{u}\right]}\end{array}$ \\
\hline$[\Lambda]$ & $\begin{array}{c}\mathrm{up}_{ア}[\Lambda \mathrm{p}] \\
\text { アップ } \\
{[\mathrm{aQpu}]}\end{array}$ & $\begin{array}{c}\text { cut }[\mathrm{k} \Lambda \mathrm{t}] \\
\text { カット } \\
{[\mathrm{kaQto}]}\end{array}$ & $\begin{array}{c}\text { pack }[\mathrm{p} \Lambda \mathrm{k}] \\
\text { パック } \\
{[\mathrm{paQku}]}\end{array}$ & - & $\begin{array}{c}\text { touch }\left[\text { tst } \int 〕\right. \\
\text { タッチ }\end{array}$ & - & $\begin{array}{l}\text { Rush } \\
\left\lceil\mathrm{r} \Lambda \int\right] \\
\text { ラッシュ } \\
\left\lceil\mathrm{raQ} \int \mathrm{u}\right]\end{array}$ \\
\hline$[0]$ & $\begin{array}{l}\text { stop 〔stop }] \\
\text { ストップ } \\
\text { 〔stoQpu〕 }\end{array}$ & $\begin{array}{c}\text { hot [hot] } \\
\text { ホット } \\
\text { [hoQto] }\end{array}$ & $\begin{array}{c}\text { knock }[\mathrm{nok}] \\
\text { ノック } \\
{[\mathrm{noQku}]}\end{array}$ & - & $\begin{array}{l}\text { watch } \\
\left.\text { [wo t } \int\right] \\
\text { ウオッチ } \\
\text { [woQ t } f \mathrm{i}]\end{array}$ & - & - \\
\hline$[u]$ & $\begin{array}{c}\text { foot [fut] } \\
\text { フット } \\
\text { [fuQto] }\end{array}$ & - & - & - & - & - & $\begin{array}{c}\text { push 〔puf〕 } \\
\text { プッシュ } \\
\left\lceil\mathrm{puQ} \int \mathrm{u}\right\rceil \\
\end{array}$ \\
\hline
\end{tabular}

Nguyên tắc chèn thêm âm ngắt cũng được áp dụng đối với các từ có một nhóm các phụ âm liền nhau đứng ở cuối và đặc biệt nhiều nhất là từ có kết thúc bằng [ks]. Ví dụ:

- [ks] $\operatorname{mix}[\mathrm{miks}] \rightarrow$クゥス [miQkusu] relax [rilæks] $\rightarrow$ リックス[riraQkusu] sex [seks] $\rightarrow$ セックス[seQkusu]

• [pl] pineapple [painæpl] $\rightarrow$ パイナップ ル [painaQpuru]

$$
\text { couple }[\mathrm{k} \wedge \mathrm{pl}] \rightarrow \text { カップル[kaQpuru }]
$$

・ [kl] tackle [tækl] $\rightarrow$ タックル[taQkuru]

・[sl] hustle [h/sl] $\rightarrow$ ハッスル[haQsuru] whistle [hwisl] $\rightarrow$ ホイッスル [hoiQsuru]

Đối với các từ 2 âm tiết, về nguyên tắc chỉ được chèn thêm âm ngắt một lần trong một từ. Ví dụ:

- pocket [pəkit] $\rightarrow$ ポケット [pokeQto]

-picnic [piknik] $\rightarrow$ ピクニック [pikuniQku]

- topic [topik] $\rightarrow$ トピック [topiQku]

\subsection{Nhật hoá nguyên âm}

Như trên đã viết, tiếng Nhật phổ thông có 5 nguyên âm là "[a] ア, [i] イ, [u] ウ, [e] エ, [o] 才”. Trong khi đó, tiếng Anh có 20 nguyên âm, và ngoài sự khác nhau giữa tiếng Anh Anh và Anh - Mỹ ra, tiếng Anh còn có nhiều phương ngữ với số lượng và tính chất của các nguyên âm trong các phương ngữ cũng tương đối khác nhau. Tuy vậy, dựa trên sự khảo sát, phân tích các âm của tiếng Anh - Anh và Anh - Mỹ cũng như mối quan hệ tương ứng khi các từ tiếng Anh du nhập vào tiếng Nhật và được Nhật hoá, các nhà Nhật ngữ học đã xác lập các nguyên tắc khi Nhật hóa các nguyên âm tiếng Anh. Cụ thể là khi tiếp nhận, xử lí bằng hệ thống âm vị của tiếng Nhật thì một vài nguyên âm có giá trị khu biệt về nghĩa trong tiếng Anh sẽ được thống nhất thành một nguyên âm trong tiếng Nhật. Rõ nét nhất là tất cả 4 nguyên âm của tiếng Anh [ $\Lambda$, ə, æ, a], đều được thống nhất thành một âm [a] của tiếng Nhật. Bảng dưới đây mô tả các trường hợp Nhật hóa nguyên âm tiếng Anh. 
Bảng 7. Các trường hợp Nhật hoá nguyên âm tiếng Anh

\begin{tabular}{|c|c|c|}
\hline STT & \begin{tabular}{|c|} 
Tiếng \\
Anh $\rightarrow$ \\
Tiếng Nhật
\end{tabular} & Ví dụ \\
\hline 1 & $\mathrm{i}: \rightarrow \mathrm{i}:$ & $\begin{array}{c}\text { cream }[\mathrm{kri}: \mathrm{m}] \rightarrow ク リ ー ム \\
{[\mathrm{kuri}: \mathrm{mu}], \mathrm{key}[\mathrm{ki}:] \rightarrow キ ー[\mathrm{ki}:],} \\
\text { meat }[\mathrm{mi}: \mathrm{t}] \rightarrow ミ ー ト[\mathrm{mi}: \mathrm{to}] \\
\text { needs }[\mathrm{ni}: \mathrm{dz}] \rightarrow \text { ニーズ[ni:zu] }\end{array}$ \\
\hline 2 & $\mathrm{i} \rightarrow \mathrm{i}$ & $\begin{array}{c}\operatorname{pink}[\text { pink }] \rightarrow \text { ピンク }[\text { pinku }], \\
\text { milk }[\text { milk] } \rightarrow \text { ミルク }[\text { miruku }], \\
\text { list }[\text { list }] \rightarrow \text { リト }[\text { risuto }]\end{array}$ \\
\hline 3 & ei $\rightarrow$ ei/ei & 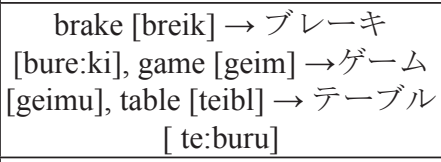 \\
\hline 4 & $\mathrm{e} \rightarrow \mathrm{e}$ & $\begin{array}{c}\text { desk [desk] } \rightarrow \text { デスク }[\text { desuku], } \\
\text { sense }[\text { sens] } \rightarrow \text { センス }[\text { sensu], } \\
\text { guest }[\text { gest] } \rightarrow \text { ゲスト }[\text { gesuto], } \\
\text { lemon }[\text { lemən] } \rightarrow \text { レモン }[\text { remon] } \\
\text { test }[\text { test] } \rightarrow \text { デスト }[\text { tesuto] }\end{array}$ \\
\hline 5 & $\begin{array}{l}æ \rightarrow \mathrm{a} \\
æ \rightarrow \mathrm{ja}\end{array}$ & 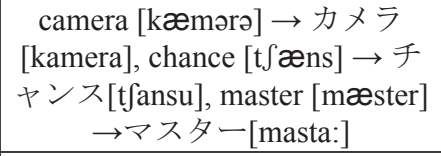 \\
\hline 6 & $\begin{array}{l}\text { ə: / ə:r } \\
\quad \rightarrow \mathrm{a}\end{array}$ & $\begin{array}{c}\text { circus [sə:rkəs] } \rightarrow \text { サーカス } \\
\text { [sa:kasu], } \\
\text { dessert [dizə:rt] } \rightarrow \text { デザート } \\
\text { [deza:to], } \\
\text { skirt [skə:rt] } \rightarrow \text { スカート } \\
\text { [suka:to] }\end{array}$ \\
\hline 7 & $\wedge \rightarrow \mathrm{a}$ & $\begin{array}{c}\text { plus }[\mathrm{pl} \wedge \mathrm{s}] \rightarrow \text { プラス }[\text { purasu }], \\
\text { puzzle }[\mathrm{p} \wedge \mathrm{zl}] \rightarrow \text { パズル }[\text { pazuru] } \\
\text { nuts }[\mathrm{n} \wedge \mathrm{ts}] \rightarrow \text { ナッツ }[\text { naQtsu] }\end{array}$ \\
\hline 8 & $\mathrm{a}: \rightarrow \mathrm{a}:$ & half [ha:f] $\rightarrow$ ハーフ[ha:fu] \\
\hline 9 & $a: / a: r \rightarrow a:$ & $\begin{array}{c}\text { card [ka:rd] } \rightarrow \text { カード[ka:do], } \\
\text { smart [sma:rt] [suma:to] }\end{array}$ \\
\hline 10 & $\mathrm{o} / \mathrm{a} \rightarrow \mathrm{o}$ & 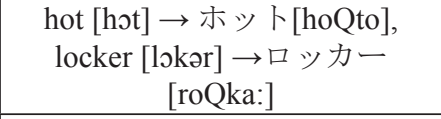 \\
\hline 11 & ๑: $\rightarrow$ o: & $\begin{array}{c}\text { call [ko:l] } \rightarrow \text { コール [ko:ru], ball } \\
\text { [bo:l] } \rightarrow \text { ボール [bo:ru] } \\
\text { corner [ko:r] } \rightarrow \text { コーナー } \\
\text { [ko:na: }] \\
\end{array}$ \\
\hline 12 & o:r $\rightarrow \mathrm{o}:$ & $\begin{array}{c}\text { report [ripo:rt] } \rightarrow \text { レポート } \\
\text { [repo:to], } \\
\text { sports [spo:rts] } \rightarrow \text { スポーツ } \\
\text { [supo:tsu] } \\
\end{array}$ \\
\hline 13 & $\mathrm{ou} \rightarrow \mathrm{o}:$ & $\begin{array}{c}\text { gold [gould] } \rightarrow \text { ゴールド } \\
\text { [go:rudo], note [nuot] } \rightarrow \text { ノート } \\
{[\text { no:to] }}\end{array}$ \\
\hline 14 & $\mathrm{u} \rightarrow \mathrm{u}$ & $\begin{array}{c}\text { full [ful] } \rightarrow \text { フル[furu], cook } \\
{[\mathrm{kuk}] \rightarrow \text { クック }[\mathrm{kuQku}]}\end{array}$ \\
\hline 15 & $\mathrm{u}: \rightarrow \mathrm{u}:$ & 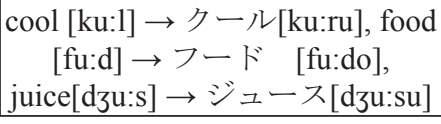 \\
\hline
\end{tabular}

\begin{tabular}{|c|c|c|}
\hline 16 & $\mathrm{ai} \rightarrow \mathrm{ai}$ & $\begin{array}{c}\text { diet [daiət] } \rightarrow \text { ダイエット } \\
\text { [daieQto], line [lain] } \rightarrow \text { ライン } \\
\text { [rain], } \\
\text { style [stail] } \rightarrow \text { スイル[sutairu], } \\
\text { guid [gaid] } \rightarrow \text { ガイド[gaido] }\end{array}$ \\
\hline 17 & $\mathrm{au} \rightarrow \mathrm{au}$ & $\begin{array}{c}\text { house [haus] } \rightarrow \text { ハウス[hausu], } \\
\text { out[aut] } \rightarrow \text { アウト[auto] }\end{array}$ \\
\hline 18 & $\mathrm{oi} \rightarrow \mathrm{oi}$ & $\begin{array}{c}\text { coin }[\text { koin }] \rightarrow \text { コイン }[\text { koin }], \text { oil } \\
[\text { oil }] \rightarrow \text { オイ } \rightarrow \text { oiru }], \\
\text { boycott }[\text { boikot }] \rightarrow \text { ボイコット } \\
{[\text { boikoQto }]}\end{array}$ \\
\hline 19 & $\begin{array}{l}\text { iə / iər } \\
\rightarrow \mathrm{i}(\mathrm{j}) \mathrm{a}\end{array}$ & $\begin{array}{c}\text { clear [kliər] } \rightarrow \text { クリアー[kuria:], } \\
\text { earing [iəringu] } \rightarrow \text { イヤリング } \\
\text { [ijaringu] }\end{array}$ \\
\hline 20 & $\begin{array}{l}\text { eə/əər } \\
\rightarrow \mathrm{i}(\mathrm{j}) \mathrm{a}\end{array}$ & $\begin{array}{c}\text { care }[\text { keər }] \rightarrow \text { ケア }[\text { kea }], \text { hear } \\
{[\text { heər }] \rightarrow ヘ ア[\text { hea }]}\end{array}$ \\
\hline 21 & $\curvearrowright / \curvearrowright ə r \rightarrow$ oa & $\begin{array}{c}\text { score }[\text { skoər] } \rightarrow \text { スコアー } \\
{[\text { sukoa], door [doər] } \rightarrow \text { ドア }[\text { doa }]}\end{array}$ \\
\hline 22 & uə/uər $\rightarrow$ ua & tour [tuər] $\rightarrow$ ツアー[tsua: \\
\hline
\end{tabular}

Tuy nhiên, có một số ngoại lệ như trong bảng dưới đây.

Bảng 8. Một số trường hợp Nhật hoá nguyên âm tiếng Anh ngoại lệ

\begin{tabular}{|c|c|c|}
\hline 1 & $\mathrm{i}: \rightarrow \mathrm{e}$ & media [mi:dər] \メディア[media] \\
\hline 2 & $\mathrm{i} \rightarrow \mathrm{i}:$ & $\begin{array}{c}\text { melody [melədi] } \rightarrow \text { メロディー[merodi: }] \\
\text { copy [kopi] } \rightarrow \text { ユピー[kopi:] }\end{array}$ \\
\hline 3 & $\mathrm{i}: \rightarrow \mathrm{e}:$ & money $[\mathrm{m} / \mathrm{ni}] \rightarrow$ マネー[mane:] \\
\hline 4 & $\mathrm{ei} \rightarrow \mathrm{e}$ & $\begin{array}{c}\text { baby [beibi] } \rightarrow \text { ベビー [bebi:], stainless } \\
\text { [steinlis] } \rightarrow \text { ステンレス [sutenresu] }\end{array}$ \\
\hline 5 & $\mathrm{ou} \rightarrow \mathrm{o}$ & poster [poustər] ↔ポスター [posuta:] \\
\hline
\end{tabular}

\subsection{Nhật hóa phụ âm và bán nguyên âm}

Khi các từ tiếng Anh du nhập vào tiếng Nhật, cũng tương tự như các nguyên âm, các phụ âm và bán nguyên âm tiếng Anh cũng được "tiếp nhận" và "xử lý” bởi hệ thống âm vị tiếng Nhật. Đối với các phụ âm [p], [b], $[\mathrm{k}]$, $[\mathrm{g}]$, vì trong tiếng Nhật có các âm tương ứng nên các phụ âm này có thể dễ dàng được thay thế bằng những phụ âm tiếng Nhật tương ứng. Tuy nhiên, tiếng Anh có số lượng phụ âm nhiều hơn tiếng Nhật nên trong quá trình Nhật hóa cũng xảy ra trường hợp có một số phụ âm tiếng Anh sẽ cùng được chuyển đổi thành một phụ âm tiếng Nhật. Kết quả là một số âm trong ngôn ngữ gốc bị mất đi khi du nhập vào tiếng Nhật, có thể thấy rất rõ điều này trong 
trường hợp âm [1] của tiếng Anh đã gần như không còn tồn tại trong các từ ngoại lai chứa âm này do [1] đã biến thành [r]. Và điều này dẫn đến hiện tượng xuất hiện nhiều từ đồng âm khác nghĩa do [1] và [r] đều trở thành $[\mathrm{r}]$. Ví dụ:

- fly [ flai] (bay) / fry [frai] (rán) $\rightarrow$ フ 个 [фrai]

- light [lait] (đèn) / right (raito) (bên phải, đúng) $\rightarrow$ ライト [raito]

Tuy nhiên, loại trừ trường hợp $[\mathrm{r}]$ ở vị trí sau các nguyên âm trong tiếng Anh khi sang tiếng Nhật gần như bị lược bỏ và được thay thế bằng [a] như một sự đánh dấu về sự tồn tại của âm $[\mathrm{r}]$ trong từ gốc tiếng Anh, nhưng [1] thì vẫn được bảo lưu trong trường hợp này. Có nghĩa là [1] và [r] khi ở vị trí này không bị thống nhất làm một. Ví dụ:

- core $[\mathrm{k} ə ə \mathrm{r}] \rightarrow コ ア[\mathrm{koa}]$

- call [ko:1] $\rightarrow コ$ ール [ko:ru]

- spare [spiər] $\rightarrow$ スペア [supea]

- spell [spel] $\rightarrow$ スペル [superu]

Ngoài ra, để có thể biểu thị một cách chính xác hơn các âm trong tiếng Anh, trong tiếng Nhật cũng hình thành những kiểu kết hợp âm mới, ngoài phạm vi các âm có trong Bảng 50 âm của tiếng Nhật. Ví dụ như $[\phi]$ ngoài kết hợp truyền thống với $[\mathrm{u}]$ tạo thành âm $[\phi \mathrm{u}]$, đã dần có khả năng kết hợp với 4 nguyên âm $[\mathrm{a}, \mathrm{i}, \mathrm{e}, \mathrm{o}]$ còn lại tạo thành các âm mới là $[\phi \mathrm{a}$, $\phi \mathrm{i}, \phi \mathrm{e}, \phi \mathrm{o}]$. Một ví dụ khác là thông thường các từ ngoại lai có âm gốc là [ti], [tu], [ti:], [tu:] đều sử dụng các âm trong hàng 夕 [ta] để chuyển âm [ti] $\rightarrow[\mathrm{t} f \mathrm{i}],[\mathrm{ti}:] \rightarrow[\mathrm{t} \mathrm{ji}:]:$

- romatic [roumæntik] $\rightarrow$ ロンチッ ク [romanchiQku]

- team $[\mathrm{ti}: \mathrm{m}] \rightarrow$ チーム $\left[\mathrm{t} \int \mathrm{i}: \mathrm{mu}\right]$
- steel [sti:1] $\rightarrow$ スチール [sutfi:ru]

- ticket [tikit] $\rightarrow$ チケット [tfi keQto]

• too [tu:1] $\rightarrow$ ツール [tsu:ru]

Tuy nhiên, đối với các từ ngoại lai du nhập vào thời kỳ sau, người Nhật đã cố gắng sử dụng cách phát âm gần với từ gốc hơn nên đã sử dụng một số kết hợp âm mới không có trong "Bảng 50 âm". Ví dụ:

- tea party [ti:pa:rti] $\rightarrow$ テイーパテー [ti:pa:ti]

• tissue [tifu:] $\rightarrow$ テッシュ [tiQsu]

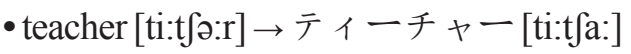

• beauty [byuti] $\rightarrow$ ビューティー [byuti:]

Bảng dưới đây thể hiện một số kiểu kết hợp âm mới hình thành trong tiếng Nhật.

Bảng 9. Các kiểu kết hợp âm mới hình thành của tiếng Nhật

\begin{tabular}{|c|c|c|c|c|c|c|}
\hline $\mathrm{Vu}$ & $\mathrm{a}(\mathrm{S})$ & i (S) & i (S) & $\mathrm{u}(\mathrm{S})$ & e $(S)$ & $\mathrm{o}(\mathrm{S})$ \\
\hline ts & $\begin{array}{c}\text { t"sa } \\
\text { ツア }\end{array}$ & $\begin{array}{l}\text { t"si } \\
\text { ツイ }\end{array}$ & $\begin{array}{l}\text { t"si } \\
\text { ツイ }\end{array}$ & - & $\begin{array}{l}\text { tse } \\
\text { ツ }\end{array}$ & $\begin{array}{l}\text { tso } \\
\text { ツオ }\end{array}$ \\
\hline $\mathrm{t}$ & & $\mathrm{ti}_{\text {イ }} \overline{\mathrm{\tau}}$ & ti & $\begin{array}{l}\text { tu } \\
\text { トゥ }\end{array}$ & & \\
\hline$\phi$ & $\phi_{\text {ア }}>$ & $\phi \mathrm{i}_{\Upsilon}>$ & $\phi i_{\Upsilon}>$ & & 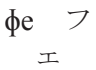 & $\begin{array}{l}\text { фо } \\
\text { フォ }\end{array}$ \\
\hline $\mathrm{j}$ & & - & - & & $\begin{array}{c}\text { je } \\
\text { 工 }\end{array}$ & \\
\hline $\mathrm{k}$ & $\begin{array}{r}\mathrm{k} \text { <wa } \\
\text { クア }\end{array}$ & $\begin{array}{l}\mathrm{k}<\mathrm{wi} \\
\text { クイ }\end{array}$ & $\begin{array}{l}\mathrm{k}<\mathrm{wi} \\
\text { クイ }\end{array}$ & - & $\begin{array}{l}\text { kwe } \\
\text { クエ }\end{array}$ & $\begin{array}{l}\text { kwo } \\
\text { クオ }\end{array}$ \\
\hline $\mathrm{W}$ & & wi ウ & wi ウ & - & $\begin{array}{l}\text { we } \\
\text { ウエ }\end{array}$ & $\begin{array}{c}\text { woウ } \\
\text { オ }\end{array}$ \\
\hline V & $\begin{array}{c}\text { va ヴ } \\
\text { ア }\end{array}$ & \begin{tabular}{c} 
vi ヴ \\
\multicolumn{1}{c}{ イ }
\end{tabular} & \begin{tabular}{c} 
vi ヴ \\
\multicolumn{1}{c}{ イ }
\end{tabular} & $\begin{array}{c}\mathrm{vu} \\
\text { ヴ }\end{array}$ & $\begin{array}{c}\text { ve ヴ } \\
\text { エ }\end{array}$ & $\begin{array}{l}\text { vo } \\
\text { ヴオ }\end{array}$ \\
\hline g & $\begin{array}{c}\text { gwa } \\
\text { グア }\end{array}$ & - & - & - & $\begin{array}{l}\text { gwe } \\
\text { グエ }\end{array}$ & - \\
\hline $\int$ & & & & & $\int e{ }^{*}$ & \\
\hline 3 & & & & & $\begin{array}{c}\text { ze } シ ゙ ~ \\
\text { エ }\end{array}$ & \\
\hline $\mathrm{t} \int$ & & \begin{tabular}{cc}
$\mathrm{t} \int \mathrm{i}$ \\
\multicolumn{1}{c}{ チ } \\
\end{tabular} & \begin{tabular}{cc}
$\mathrm{t} \int \mathrm{i}$ \\
\multicolumn{1}{c}{ チ } \\
\end{tabular} & & $\begin{array}{c}\mathrm{t} \int \mathrm{e} \text { チ } \\
\text { 工 }\end{array}$ & \\
\hline dz & & & & & $\begin{array}{l}\text { dze } \\
\text { ヂエ }\end{array}$ & \\
\hline $\mathrm{t}$ & - & - & - & $\frac{\text { tju }}{\bar{\tau}_{\text {一 }}}$ & - & - \\
\hline
\end{tabular}




\begin{tabular}{|c|c|c|c|c|c|c|}
\hline $\mathrm{d}$ & - & - & - & $\begin{array}{c}\text { dju } \\
\text { デュ }\end{array}$ & - & - \\
\hline$\phi$ & - & - & - & $\begin{array}{c}\phi j u フ \\
\text { ユ }\end{array}$ & & \\
\hline $\mathrm{v}$ & - & - & - & $\begin{array}{c}\text { Vjuヴ } \\
\text { ユ }\end{array}$ & - & - \\
\hline
\end{tabular}

Tương tự, các âm [di], [di:] trong âm gốc tiếng Anh nếu ở trong những từ ngoại lai du nhập sớm vào tiếng Nhật đều được thay thế bằng [dzi] và [dzi:] khi đứng ở đầu từ hoặc bằng âm [zi], [zi:] nếu đứng ở giữa từ, ví dụ:

- credit [kredit] $\rightarrow$ クレジット [kureziQto]

Các từ du nhập sau này chủ yếu sử dụng âm [di], [di:]. Ví dụ:

• dealer [di:lər] $\rightarrow$ デイーラー [di:ra:]

・ handicap [hændikæp] $\rightarrow$ ハンディキ ヤップ [handikjaQpu]

・ wedding [wedin] $\rightarrow$ ウエデイング [uediNgu]

- melody [melodi] $\rightarrow$ メロデイー [merodi:]

・ dinner [dinər] $\rightarrow$ ディナー [dina:]

\subsection{Nhật hóa theo cách đánh vần của tiếng Nhật}

Các nguyên âm trong âm tiết không có trọng âm ở từ tiếng Anh khi Nhật hóa đều được biến đổi dựa theo cách đánh vần của tiếng Nhật. Chính vì vậy, nếu nhìn từ góc độ ngữ âm, các từ được Nhật hóa theo cách này có cách phát âm rất khác so với cách phát âm của từ gốc, và nhiều khi rất khó nhận biết từ. Dưới đây là một số trường hợp điển hình:

\section{a. "ij"}

Trong tiếng Anh, âm vị này được viết là “i”, nhưng có nhiều cách phát âm khác nhau: có trường hợp vẫn đọc là [i] như các từ dưới đây và trong từ được Nhật hóa cũng đọc là [i], theo cách đánh vần của tiếng Nhật .
・ service [sə:rvis] $\rightarrow$ サービス [sa:bisu]

- pink $[\mathrm{pi \eta k}] \rightarrow$ ピンク [piNku]

Khi âm này không được đặt trong âm tiết có trọng âm thì được phát âm thành [ə], nhưng khi sang tiếng Nhật vẫn được phát âm thành [i], như cách đánh vần. Ví dụ:

- animation [ænəmeifn] $\rightarrow$ アニメーシ ヨン [anime: fon ]

- delicate [deləkit] $\rightarrow$ デリケート [derike:to]

b. "e"

Trong các từ tiếng Anh dưới đây, "e" được đọc là [ə], nhưng khi chuyển sang tiếng Nhật "e" vẫn được phát âm theo kiểu đánh vần tiếng Nhật là [e]:

- cancel [kænsəl] $\rightarrow$ キャセル [kjaNseru]

- camera [kæmərə] $\rightarrow$ カ メラ [kamera]

Dưới đây là một số ví dụ khác về các từ tiếng Anh được Nhật hoá theo cách đánh vần:

・dam[dæm] $\rightarrow$ ダ ム [damu $]$

- instant [instənt] $\rightarrow$ イスタント [insutaNto]

• calory [kæləri] $\rightarrow$ カリー[karori:]

- lemon [lemən] $\rightarrow$ モン [remon]

- piano [piænou] $\rightarrow$ ピアノ [piano]

\subsection{Nhật hoá trọng âm}

Có sự khác nhau rất lớn giữa trọng âm tiếng Anh và tiếng Nhật: Nếu như ở tiếng Anh trọng âm được phân biệt bởi sự khác nhau về độ mạnh yếu giữa các âm tiết, thì ở tiếng Nhật là sự khác nhau về độ cao thấp của các phách. Và đặc biệt, trong một từ tiếng Nhật thì giữa phách thứ nhất và phách thứ hai luôn khác nhau về độ cao thấp, tức là nếu phách thứ nhất 
là cao thì phách thứ hai sẽ phải thấp và ngược lại. Ví dụ:

Bảng 10. Mô hình trọng âm trong từ tiếng Anh đã được Nhật hóa

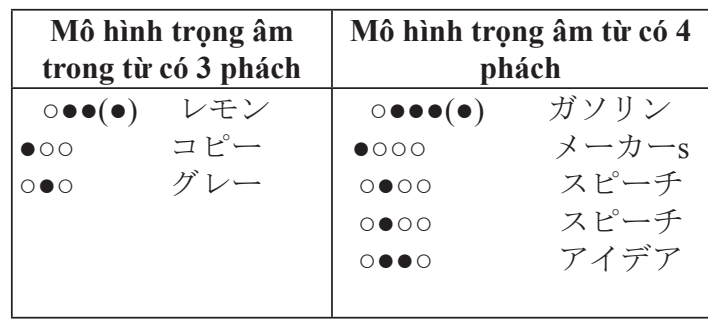

(Ghi chú: •: cao, ○: thấp, () ngũu điệu của trợ tì )

Điểm khác biệt mang tính bản chất về trọng âm giữa hai ngôn ngữ này đã tạo nên sự khác nhau nhất định của trọng âm trong từ gốc tiếng Anh và từ đã Nhật hoá. Đối với phương thức Nhật hóa bằng trọng âm, có một số nguyên tắc như sau:

Đối với các âm tiết được đặt trọng âm trong từ gốc tiếng Anh thì các phách tương ứng được phát âm cao trong từ tiếng Nhật. Ngoài ra, nếu trong từ gốc, âm tiết có trọng âm là nguyên âm dài hoặc nguyên âm đôi thì khi chuyển sang tiếng Nhật bắt buộc phải chuyển thành hai phách và trong trường hợp này, chỉ phách đứng trước được coi là trung tâm của trọng âm mà thôi. Ví dụ:

- cream ['kri:m] $\rightarrow$ クリム $[\mathrm{ku}$ ri :mu]

- tournament [tu'ərnəmnət] $\rightarrow$ トーナメ ント[ $[$ to : name nto])

Tuy nhiên, trong tiếng Nhật cũng có những từ ngoại lai tiếng Anh không có trọng âm, tức là các phách trong từ đó được phát âm với độ cao như nhau mặc dù từ gốc tiếng Anh có trọng âm:

- lemon [le'mən] $\rightarrow$ モン

・ catalogue [kæ’təlog] \カタログ
• table [teibl] $\rightarrow$ テーブル

- minus [ma'inəs] $\rightarrow$ マイナス

・violin [vaioli’n] $\rightarrow \quad$ バイオリン

\section{Kết luận}

Trên đây, trên cơ sở miêu tả một số đặc điểm của ngữ âm tiếng Nhật và phân tích một số nét khác biệt của ngữ âm tiếng Anh so với ngữ âm tiếng Nhật, các tác giả đã làm rõ những nét cơ bản của 6 phương thức Nhật hóa từ gốc tiếng Anh trong tiếng Nhật về mặt ngữ âm. Cũng như từ ngoại lai trong các ngôn ngữ khác, các từ tiếng Anh khi du nhập vào tiếng Nhật để trở thành từ ngoại lai của tiếng Nhật đòi hỏi phải thực hiện một số "thao tác" nhất định nhằm chuyển đổi phát âm và chữ viết cho phù hợp với tiếng Nhật, nhưng do tiếng Nhật không thuộc hệ ngôn ngữ La tinh và có những đặc trưng riêng về ngữ âm (ví dụ như số lượng nguyên âm ít, hầu hết âm tiết là âm tiết mở, có đơn vị phách/ mora, trọng âm cao thấp v.v..) nên sự chuyển đổi này phức tạp hơn và tạo nên nhiều sự thay đổi rõ rệt so với từ gốc. Điều đó nhiều khi gây khó khăn cho người sử dụng tiếng Nhật, ngay cả đối với những người sử dụng tiếng Nhật biết tiếng Anh, có nhiều trường hợp khó "liên hệ" được từ ngoại lai gốc tiếng Anh trong tiếng Nhật với vỏ ngữ âm của từ gốc và thậm chí người bản ngữ tiếng Anh cũng không nhận ra một số từ khi nghe người Nhật phát âm

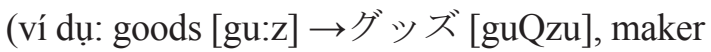
[meikə] $\rightarrow$ メカー[me:ka:]). Với lý do đó, các tác giả hi vọng rằng bài viết đã phần nào khắc họa được những nguyên tắc chung trong việc Nhật hóa từ gốc tiếng Anh trong tiếng Nhật, giúp những người sử dụng tiếng Nhật dễ dàng hơn trong việc nhận biết và sử dụng nhóm từ này. Tuy nhiên, từ ngoại lai trong tiếng Nhật nói chung và từ ngoại lai gốc tiếng Anh trong tiếng Nhật nói riêng là một đề tài rộng, việc 
Nhật hóa từ ngoại lai gốc tiếng Anh nói chung và Nhật hóa từ ngoại lai gốc tiếng Anh từ góc độ ngữ âm nói riêng cũng là vấn đề phức tạp, nhiều trường hợp ngoại lệ mà trong phạm vi bài viết này chúng tôi chưa thể đề cập hết được. Những nội dung còn lại của đề tài này chúng tôi sẽ tiếp tục nghiên cứu và trình bày trong các bài viết tiếp theo.

\section{Tài liệu tham khảo}

\section{Tiếng Việt}

Nguyễn Quang Hồng (2002). Âm tiết và loại hình ngôn ngũ. NXB Đại học Quốc gia Hà Nội.

Trần Kiều Huế (2007). Một số đặc điểm của tì ngoại lai tiếng Anh trong tiếng Nhật, Kỉ yếu Hội thảo quốc tế về giảng dạy tiếng Nhật, Nxb Đại học Quốc gia Hà Nội, tr.157-165.

Nguyễn Văn Khang (1999). Ngôn ngũ học xã hội Nhũng vấn đề co bản. NXB Khoa học Xã hội.

Nguyễn Văn Khang (2007). Tù ngoại lai trong tiếng Việt. NXB Khoa học Xã hội.
Đoàn Xuân Kiên (1998). Xem lại một vấn đề ngũ âm tiếng việt: cấu trúc âm tiết. Hợp Lưu 48. Bỉ.

Ngô Minh Thủy (2005). Tù ngoại lai trong tiếng Nhật. Tạp chí Khoa học, 2005, số 4.

\section{Tiếng Anh}

Peter Roach (1988). English Phonetics and Phonology. Cambridge University Press.

\section{Tiếng Nhật}

青木健一日下洋石 (1990)、『現代カタカナ用 語辞典』、日本文芸.

秋元美晴、(2003)、『よくわかる語彙』、ア ルク。

石野博史、(1983) 、『現代外来語考』、大修 館書店.

石綿 敏雄、(2001)、『外来語の総合的研究』 、東京堂出版.

国際交流基金一日本語国際センター（1995） 、『発音』、凡人者.

国際交流基金一日本語国際センター（1995） 、『日本語への招待』、凡人者.

国立国語研究所 (加ッケンブッシュ寬子) 、(1990)、『外来語の形成とその教育』

\title{
METHODS OF JAPANIZING BORROWING WORDS OF ENGLISH ORIGIN IN JAPANESE LANGUAGE (FROM PHONETIC PERSPECTIVE)
}

\author{
Ngo Minh Thuy, Tran Kieu Hue
}

\begin{abstract}
Faculty of Japanese Language and Culture, VNU University and Languages and International Studies, Pham Van Dong, Cau Giay, Hanoi, Vietnam
\end{abstract}

\begin{abstract}
Foreigners studying Japanese language, especially those who can speak English might find it difficult to learn borrowing words of English origin. This results from the fact that when English words were borrowed into Japanese, they were so enormously modified particularly regarding their sounds and letters that they can hardly be recognized even by native speakers of English. In this article, based on an overview of phonetics, written Japanese and the basic differences between English and Japanese phonetics, the authors analyze the methods of Japanizing words of English origin from phonetic perspective to help learners of Japanese understand some rules of Japanizing words of English origin so that they can identify and use Japanese words of English origin more easily.
\end{abstract}

Keywords: words of foreign origin, borrowing words of English origin, methods of Japanizing words, phonetics 